\title{
Nanoscale Strain and Composition Mapping in Ionic Thin Film Heterostructures for Resistive Switching Devices
}

\author{
William J.Bowman ${ }^{1,2}$, Sebastian Schweiger ${ }^{2}$, Amith Darbal $^{3}$, Peter Crozier $^{1}$, Jennifer L.M. Rupp ${ }^{2}$
}

1. School for the Engineering of Matter, Transport and Energy, Arizona State University, USA

2. Electrochemical Materials Group, ETH Zürich, Zürich, Switzerland

3. AppFive LLC, Tempe, AZ, USA

Interfacial strain control of electrical conductivity and resistive switching behavior in multilayer thin film oxide heterostructures has recently been reported for $\mathrm{Gd}_{0.1} \mathrm{Ce}_{0.9} \mathrm{O}_{2-\delta} \mid \mathrm{Er}_{2} \mathrm{O}_{3} \quad(\mathrm{GCO} \mid \mathrm{ERO})$ heterostructures fabricated using pulsed laser deposition [1]. Strain control was achieved by growing alternating layers of insulating and conducting oxides (fig. 1a,b) whose dissimilar lattice constants result in interfacial lattice mismatch. Total strain in the conducting layers (i.e. GCO) is modulated via choice of insulating oxide and/or total interface count at constant device thickness (fig. 1a,b shows $275 \mathrm{~nm}$ tall devices with 6 and 60 interfaces, respectively). Furthermore, depending on the composition of the insulating phase, tensile or compressive strain may be imparted on the conducting GCO. Here we employ precession-enhanced nanodiffraction (PEND) and electron energy-loss spectroscopy (EELS) in an aberration-corrected STEM to assess the nanoscale strain and compositional distribution in layers of a strained heterostructure microdevice.

An initial analysis of the biaxial strain state of compressively-strained GCO in GCO-ERO heterostructure devices has been performed using diffractograms like that in fig. 1c, computed within the GCO layers from atomic resolution TEM images, e.g. fig. 2a,c. The mean in-plane [311] and out-ofplane [111] Miller spacing in the 6 and 60 interface samples were each determined from 4 to 6 measurements of their reciprocal-lattice vectors (from most intense pixel in diffraction spot). In the 6 interface sample the Miller spacing was measured in two regions: The first directly adjacent to the GCOERO interface (region " 1 " in fig. 2a), the second a distance $12 \mathrm{~nm}$ from the GCO-ERO interface (region " 2 " in fig. 2a). Miller spacing in the 60 interface sample was measured over the entire GCO layer (region " 1 " in fig. 2c). From this we observe that the GCO out-of-plane Miller spacing ([111]) is greatest near the GCO-ERO interface in the 6 interface samples (i.e. region " 1 " in fig. 1a), and that the in-plane spacing is smallest, indicating biaxial compressive strain on the GCO at the interface. Away from the interface, however, e.g. region " 2 " in fig. 1a, the [111] and [311] spacings are relatively smaller and larger, respectively, suggesting that the interfacial strain field has dissipated and the GCO lattice is more relaxed. Additionally, in the 60 interface sample, the strain state is similar to the near-interface region of the 6 interface sample; i.e. when averaged over the layer, the GCO lattice in the 60 interface sample appears fully strained.

In this contribution, we extend this Fourier analysis of the heterolayer strain state by employing a probecorrected JEOL ARM200F equipped with an ASTAR PEND system capable of detecting relative lattice constant changes with nanometer spatial resolution from thousands of nanodiffraction patterns [2], and will be applied to determine the strain distribution across layers, at conductor-insulator interfaces and in grain boundary regions. These data are correlated with spatially-resolved compositional and chemical data acquired via STEM EELS performed using the same aberration-corrected instrument. Together, these nanoscale strain, composition and chemical analyses are interpreted in terms of microscopic transport measurements of current-voltage characteristics and AC impedance. Finally, the influence of insulating material composition - and the resultant degree of compressive or tensile strain imparted on 
the conducting phase, is discussed in light of a studies performed on strained heterolayers with different insulating oxides [3].

References:

[1] S. Schweiger, et al., ACS Nano 8:5 (2016), p. 5032.

[2] A. Darbal, et al., Microsc. \& Microanal. 19:S2 (2013), p. 702.

[3] We acknowledge ScopeM at ETH Zürich and the John M. Cowley Center for High Resolution EM at ASU, and thank the staff for their support. Work was supported by the Swiss NSF (project numbers 155986 and 138914). W.J.B. was a Swiss Government Excellence Scholarship holder for the academic year 2015-2016 (ESKAS 2015.1183); and acknowledges financial support of the US NSF Graduate Research Fellowship Program (DGE-1311230), and NSF DMR-1308085.

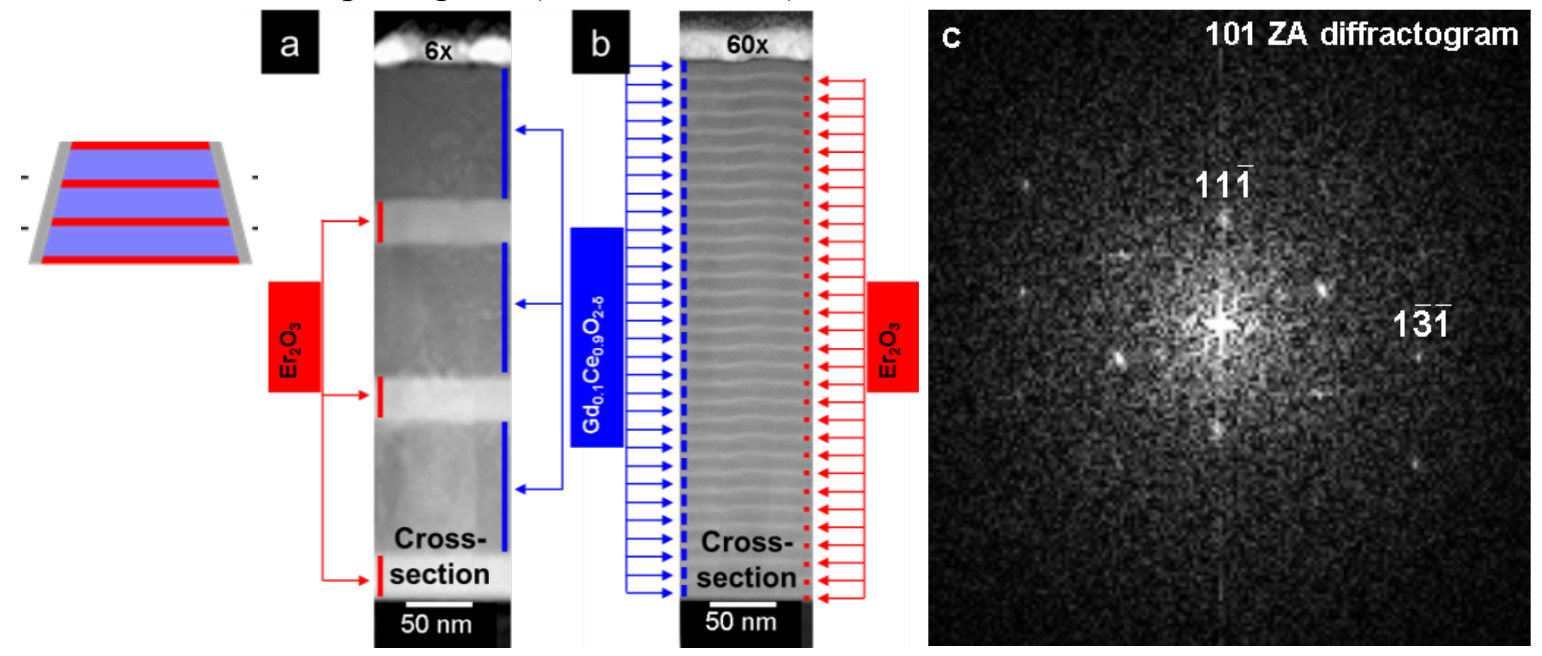

Figure 1. $(a, b)$ Schematic of 6 interface multilayer sample and cross section ADF STEM (FEI Talos) images of 6 and 60 interfaces samples, respectively. (c) Typical diffractogram from GCO.

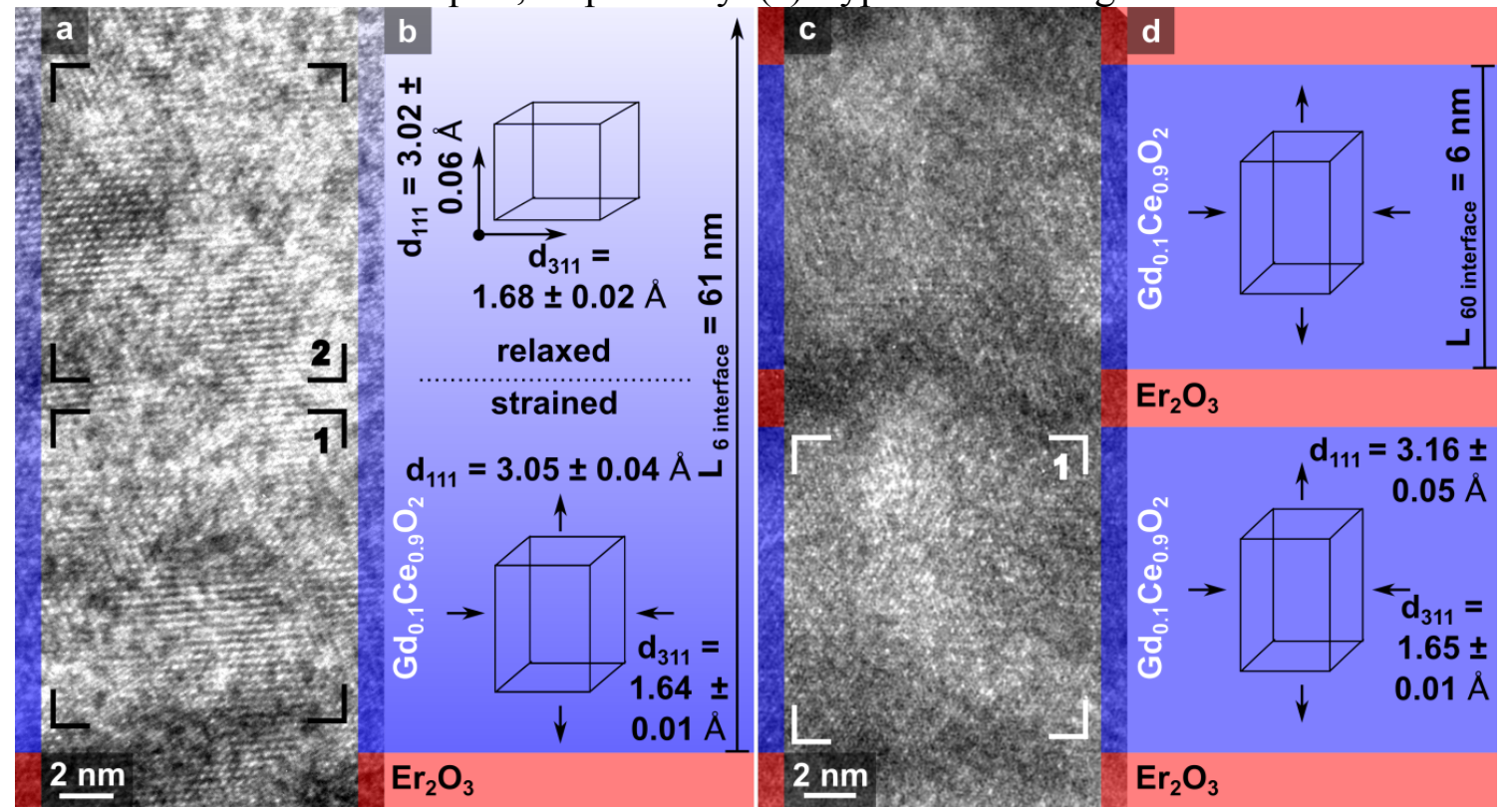

Figure 2. (a,c) BFTEM images of GCO-ERO interface(s) in 6 and 60 interface samples, respectively (FEI Tecnai F30). Regions labelled "1" and "2" indicate the areas used to compute diffractograms. (b,d) Diagram illustrating the strain state in the conducting oxide phase and measured Miller plane spacing. 